\title{
Identifying leatherback turtle foraging behaviour from satellite telemetry using a switching state-space model
}

\author{
Ian D. Jonsen*, Ransom A. Myers ${ }^{\mp}$, Michael C. James \\ Department of Biology, Dalhousie University, 1355 Oxford Street, Halifax, Nova Scotia B3H 4J1, Canada
}

\begin{abstract}
Identifying the foraging habitat of marine predators is vital to understanding the ecology of these species and for their management and conservation. Foraging habitat for many marine predators is dynamic, and this poses a serious challenge for understanding how oceanographic features may shape the ecology of these animals. To help resolve this issue, we present a switching state-space model (SSSM) for discerning different movement behaviours hidden within error-prone satellite telemetry data. Along with modelling the movement dynamics, the SSSM estimates the probability that an animal is in a particular discrete behavioural mode, such as transiting or foraging. Using Argos satellite telemetry for leatherback sea turtles, we show that the SSSM readily identifies distinct classes of movement behaviour from the noisy data. Moreover, patterns in simultaneously collected diving data, to which the model is blind, match well with behavioural mode estimates. By combining behavioural mode estimates from the model with the diving data, we show that while transiting, leatherbacks make longer, deeper dives; and while foraging, they encounter cooler waters that range from 13 to $22^{\circ} \mathrm{C}$. These differences are consistent among the turtles studied and within the same turtle in different years. This modelling approach can enhance standard kernel density estimators for identifying habitat use by incorporating behavioural information into the estimation procedure. Ultimately, we can build predictive models of habitat use by incorporating environmental data and diving behaviour directly into the SSSM framework.
\end{abstract}

KEY WORDS: Bayesian - Correlated random walk - Dermochelys coriacea - Diving behaviour . Habitat $\cdot$ Hidden Markov models $\cdot$ Meta-analysis $\cdot$ Uncertainty

\section{INTRODUCTION}

Electronic tracking of marine predators has revealed a wealth of information on patterns of distribution and habitat use (Block et al. 2005), migratory patterns (Block et al. 2001), and foraging ecology (Sale et al. 2006). We contend, however, that even more information can be extracted from these data by applying modern statistical methods that can deal with both biological and statistical complexity in the data (Jonsen et al. 2005, Nielsen et al. 2006) and allow estimation of hidden processes that are intractable to other approaches.

Several approaches have been recently developed that allow estimation of hidden states from time series data. State-space models have great potential for modelling population time series data and have been generalized to admit a variety of population data types and analyses (Newman et al. 2006). The state-space approach has also been proposed as a powerful tool for modelling animal movement data because of its ability to deal simultaneously with potentially large measurement errors and variability in the dynamics of movement (Jonsen et al. 2003). Hidden Markov models, unlike state-space models, do not estimate dynamics, but they can identify hidden patterns in the data. This was essentially the approach used by Morales et al. (2004), who analysed GPS telemetry on elk movements and showed how a switching model could be used to reveal long- and short-range movement behaviours 
hidden in the data. Jonsen et al. (2005) further developed the switching idea by showing how different behaviours that were obscured by measurement error in Argos satellite telemetry data could be estimated using a state-space approach that modelled the movement dynamics and accounted for uncertainty in both the observations and the dynamics. The fundamental advantage of the state-space approach is that movement dynamics are modelled explicitly and this allows more complex behaviour to be captured, even when the issue of measurement error is minor, such as with GPS data.

Here, we consider the application of a switching state-space model (SSSM) to the issue of identifying foraging behaviour from remotely sensed tracking data-a situation where direct observation is not possible. When animals encounter areas of sufficiently abundant prey, they often engage in area-restricted search by decreasing their travel rate and/or increasing their turning frequency and angle (Turchin 1991). Conversely, animals encountering unsuitable habitats often have fast travel rates and infrequent and small turning angles (Turchin 1991). These differences in movement behaviour can form the basis for estimating nominal foraging and transiting behaviours from a time series of observed positions. To identify different behaviours that may be hidden in the position data, we employ an SSSM. The purpose of an SSSM is to estimate: (1) unobservable 'true' positions, termed state estimates; (2) movement parameters from a specified process model; and (3) hidden behavioural states from data observed with error. Hereafter we refer to these behavioural states as behavioural modes to avoid confusion with the state estimates.

We illustrate our approach by fitting an SSSM to Argos-derived positions of leatherback turtles off the coasts of eastern Canada and the northeastern United States. The leatherback turtle Dermochelys coriacea is a cosmopolitan marine species that undertakes a variety of migratory patterns (Luschi et al. 2003, James et al. 2005a). Leatherbacks tagged off Nova Scotia, Canada, make annual return migrations from tropical to sub-Arctic waters (James et al. 2005a), with much of their time in northern waters presumably spent foraging on gelatinous zooplankton (James et al. 2006a). Despite a growing awareness of the migratory strategies and behaviours (James et al. 2005a, Jonsen et al. 2006) and population characteristics (James et al. 2007) of these animals, we know remarkably little about how they utilize foraging habitat, and even less about the ecology of their jellyfish prey (Witt et al. 2007). Efforts to identify habitats important to this species (James et al. 2005b) are urgently needed, given its critically endangered status (IUCN 2004). To this end, we illustrate how SSSMs can be used to estimate locations where turtles are foraging based solely on the information contained in the time series of their observed positions. Then, as a means of corroborating the SSSM predictions, we compare estimated switches between behavioural modes to observed changes in patterns of diving activity that were collected simultaneously. Finally, using all the suitable telemetry data available to us, we describe differences in diving behaviour and the thermal environment of leatherback turtles that engage in foraging versus transiting behaviours.

\section{MATERIALS AND METHODS}

Data. The data consist of Argos-derived surface positions obtained from satellite-linked time-depth recorders (SLTDRs; model SSC3, Wildlife Computers) attached to leatherback turtles captured in waters off Nova Scotia, Canada (see James et al. 2005b for details). We focus our analyses on movement behaviours and diving data in the northeastern Atlantic $\left(>36^{\circ} \mathrm{N}\right)$ off eastern North America, where the turtles spend considerable time foraging (James et al. 2005b).

In addition to providing surface positions, the SLTDRs collect and relay data on time at depth, time at temperature, maximum dive depth and dive duration. These dive data are not directly associated with the recorded positions, rather they are binned within 14 user-defined data ranges over $6 \mathrm{~h}$ collection periods. Periods were set such that one consistently encompassed night and one encompassed day (Night: 21:0003:00 h; Morning: 03:00-09:00 h; Day: 09:00-15:00 h; Evening: 15:00-21:00 h; Atlantic Daylight Time). Time at depth and time at temperature reflected all time when SLTDRs were submerged, whereas dives were registered only when turtles descended below $4 \mathrm{~m}$ or $6 \mathrm{~m}$ (depending on the tag used). These data are transmitted in histogram format to increase ease of transfer via the limited bandwidth of the Argos satellite system. Nonetheless, patterns in diving behaviour can be readily identified and compared with the behavioural estimates from the SSSM described below.

Suitable data were available for 5 turtles, 2 of which were deployed with tags set to record continuously, and 3 with tags that were duty-cycled (set to record every second $24 \mathrm{~h}$ period). Leatherbacks in these northern waters complete annual return migrations from tropical waters in the Caribbean or South America (James et al. 2005a) and 4 of the 5 tags (3 dutycycled, 1 continuously recording) transmitted long enough to record parts of 2 successive seasons spent foraging in northern waters. We consider only those portions of the data north of $35^{\circ}$ Lat. where the turtles appear to forage extensively. The duration of observed tracks ranged from 49 to $216 \mathrm{~d}$ (mean $124 \mathrm{~d} \pm 46 \mathrm{SD}$ ). 
Switching state-space model. Here, we describe the components of the SSSM employed to estimate hidden behavioural modes, states, and movement parameters from the Argos satellite telemetry data.

We use a first-difference correlated random walk model (DCRW) (Jonsen et al. 2005) to model the movement process. The DCRW assumes a correlated random walk on the differences in successive locations, not on the locations themselves. This makes sense intuitively as the behaviour we are interested in is how animals change their speed and direction, not how they change their location per se. Written as a statespace process model in 2 dimensions, the DCRW has the following form,

$$
\mathbf{d}_{t} \sim N_{2}\left[\gamma \mathbf{T}(\theta) \mathbf{d}_{t-1}, \boldsymbol{\Sigma}\right]
$$

where $\mathbf{d}_{t-1}$ is the difference between the locations $\mathbf{x}_{t-1}$ and $\mathbf{x}_{t-2}$, and $\mathbf{d}_{t}$ is the difference between the locations $\mathbf{x}_{t}$ and $\mathbf{x}_{t-1} . \mathbf{T}(\theta)$ is a transition matrix that provides the rotation required to move from $\mathbf{d}_{t-1}$ to $\mathbf{d}_{t}$, where $\theta$ is the mean turning angle. $N_{2}$ is a bivariate Gaussian distribution with covariance matrix $\boldsymbol{\Sigma}$. We include $\gamma$ to allow for variability in the autocorrelation of direction and speed; with $\gamma=0$ resulting in a simple random walk and $0<\gamma<1$ yielding a random walk with correlation in both direction and move speed.

The second component of the state-space model relates the unobserved states predicted by the process model to the observed data; consequently it is termed the observation model. Rather than use a simple model where each unobserved state corresponds to an observed location, we must account for the irregular sampling of positions, the variable quality of Argos observations, and their non-Gaussian errors (see Jonsen et al. 2005 for full details).

We let $i$ be an index for locations (if any are observed) between time $t$ and $t+1$, i.e. $i=\left(0, \ldots, n_{t}\right)$. We make the simplifying assumption that animals travel in a straight line between $\mathbf{x}_{t-1}$ and $\mathbf{x}_{t}$. This poses no difficulty for state transitions with reasonably short time steps, relative to the resolution of the data,

$$
\mathbf{y}_{t, i}=\left(1-j_{i}\right) \mathbf{x}_{t-1}+j_{i} \mathbf{x}_{t}+\boldsymbol{\varepsilon}_{t}
$$

where $\mathbf{y}_{t, i}$ is the $i$ th observed position during the regular time interval $t-1$ to $t, j_{i}$ is the proportion of this time interval at which the $i$ th observation is made $\left(0<j_{i}<1\right)$, and $\boldsymbol{\varepsilon}_{t}$ is a random variable representing the error in the Argos-derived positions. Note that $j_{i}$ can be calculated from the data if the time of day is recorded with each observed location, and that for regular time intervals where no observations exist we set $i=1$ and $j_{i}=0.5$. Because Argos position errors can be strongly nonGaussian (Jonsen et al. 2005), we model the errors with generalized $t$-distributions which are robust to extreme values. In addition, Argos categorizes positions into
6 quality classes and we use this information to determine the appropriate $t$-distribution parameters to use for each position in the observation model. For estimation errors in latitude or longitude of quality class $q(q=$ $1, \ldots, 6)$ we let $\boldsymbol{\varepsilon}_{q(i), t} \sim t\left(0, \tau_{q(i), t}, \mathbf{v}_{q(i), t}\right)$, where $\tau_{q(i), t}$ is the scale parameter and $\boldsymbol{v}_{q(i), t}$ is the degrees of freedom. When fitting the state-space model to the Argos-derived position data, we fix $\boldsymbol{\varepsilon}_{t}$ to values estimated from independent data (Jonsen et al. 2005), thereby avoiding the need to estimate an additional 24 parameters.

With the state-space model described above we can estimate the unobserved states $\mathbf{x}_{t}$ and the parameters of the DCRW model, $\theta, \gamma$ and $\boldsymbol{\Sigma}$. We still require a method to identify discrete behavioural modes from the position data. To do this, we specify a process model for each behavioural mode we believe exists in the data, i.e. we use an index $b_{t}$ to denote behavioural mode on the parameters $\theta$ and $\gamma$ (Morales et al. 2004). Thus Eq. (1) becomes:

$$
\mathbf{d}_{t} \sim N_{2}\left[\gamma_{b_{t}} \mathbf{T}\left(\theta_{b_{t}}\right) \mathbf{d}_{t-1}, \boldsymbol{\Sigma}\right]
$$

where $b_{t}=k, k \in\{1, \ldots, B\}$ and $B$ is the total number of behavioural modes to be estimated. Here we consider 2 general behaviours, nominally, transiting and foraging. This is the DCRWS model (from Jonsen et al. 2005).

The analysis now involves the estimation of 2 sets of unobserved variables, $\mathbf{x}_{t}$ and $b_{t}$ and the estimation of the parameters $\theta, \gamma$, and $\boldsymbol{\Sigma}$. We can estimate $b_{t}$ using a mixture model approach, which assumes that what an animal is doing now is independent of what it was doing previously. However, a more realistic approach is to assume that what an animal is doing now depends to some extent upon what it was doing previously. With this in mind, we make use of a switching model (Morales et al. 2004, Jonsen et al. 2005) where the movement parameters $\theta$ and $\gamma$ are indexed by behavioural mode. In our case, we are interested in transiting and foraging behaviours, so there are 4 possible transitions, and we must estimate 2 of these. We let $\alpha_{1}$ be the probability of an animal transiting at time $t$, given it was also transiting at time $t-1$, and we let $\alpha_{2}$ be the probability of an animal transiting at time $t$, given it was foraging at time $t-1$.

We use a Bayesian approach to fit the model, placing vague priors on model parameters (see Jonsen et al. 2005 for details), except for the movement parameters for the transiting mode: $\theta_{1}$ and $\gamma_{1}$. For these parameters we used the following priors: $\theta_{1} \sim \operatorname{Beta}(20,20)$ and $\gamma_{1} \sim \operatorname{Beta}(48,16)$; reasoning that while transiting, turn angles should be closer to 0 and autocorrelation should be higher than when foraging. The model was fit with the freely available WinBUGS software (Spiegelhalter et al. 2004) which uses Markov Chain Monte Carlo (MCMC) methods to approximate the multi-dimensional integration required in Bayesian analyses. The model 
was fit to each dataset with a total of $30000 \mathrm{MCMC}$ samples, the first 10000 were discarded as a burn-in and the remaining 20000 samples were thinned out to 4000 samples by retaining only every 5 th sample to reduce autocorrelation. Parameter, state, and behavioural mode estimates were based on these final 4000 samples. The model code is provided in Appendix 1 (available in MEPS Supplementary Material at www.int-res/articles/ suppl/m337p255_app.pdf ).

Analyses. We used the diving statistics described earlier, which show relatively clear behavioural patterns (James et al. 2006a), as a means of corroborating the estimates of $b_{t}$ obtained from the DCRWS model. Because $b_{t}$ is a discrete parameter-values can only be 1 or $2-$ we used the means of the MCMC samples as a convenient way to visualize behavioural switches. We, therefore, delineated the 2 behavioural modes by adopting cut-offs at 1.25 and 1.75 ; mean estimates below 1.25 were considered to represent transiting and mean estimates above 1.75 were considered to represent foraging. Mean estimates between 1.25 and 1.75 were treated as uncertain, i.e. there was insufficient information to distinguish between the behaviours in these cases. We view this as a conservative approach to classifying the behaviour modes as one could easily assume a single cut-off of 1.5 .

We used a graphical approach, overlaying a time series histogram of binned diving statistics (14 data ranges in $6 \mathrm{~h}$ collection intervals) with $b_{t}$ (also at $6 \mathrm{~h}$ intervals) to look for congruence between estimated switches in $b_{t}$ and obvious changes in the temporal pattern of the diving statistics. We also aggregated the data over time and compared the proportion of dives that occurred while turtles were predicted to be transiting or foraging within each of the 14 data ranges. Finally, for each diving statistic we present plots of the difference in the mean for time periods when the turtle was transiting versus foraging. The means of the diving statistics were calculated from time series data, and we accounted for autocorrelation by estimating the effective sample size (Bayley \& Hammersley 1946) and adjusting the df for the confidence intervals. The differences between transiting versus foraging for temperature, time at depth, dive duration and maximum dive depth were each combined using a random effects meta-analysis (Worm \& Myers 2003). As mentioned earlier, 4 of the tags provided data on successive foraging seasons in northern waters. We consider these multiple foraging seasons to be distinct enough to consider them as independent datasets in this analysis. We do, however, evaluate the more conservative assumption of nonindependence among tracks from the same turtle.

\section{RESULTS}

Maps of state estimates with associated behavioural modes (Fig. 1 \& Fig. A2-1 [Appendix 2 available at: www.int-res.com/suppl/m337p255_app.pdf) indicate that 2 of the turtles spent a considerable proportion of time $\left(0.74\right.$, Turtle $A_{i} 0.55$, Turtle B.1) foraging in shelf (Fig. 1, red circles) or slope (Fig. A2-1, red circles) waters off Canada and the northeastern United States. Estimated foraging bouts for the other turtles occurred in waters from 37 to $50^{\circ} \mathrm{N}$ with most bouts occurring in slope waters south and east of George's Bank, the southern Gulf of St. Lawrence, and off northeastern Cape Breton Island. The proportion of time spent foraging in northern waters by these turtles ranged from 0.28 to 0.76 .

There was relatively little uncertainty in the behavioural mode, as less than $10 \%$ of state estimates had behavioural modes estimated between 1.25 and 1.75 (Fig. 1, black circles). These uncertain behavioural modes were associated either with short transition

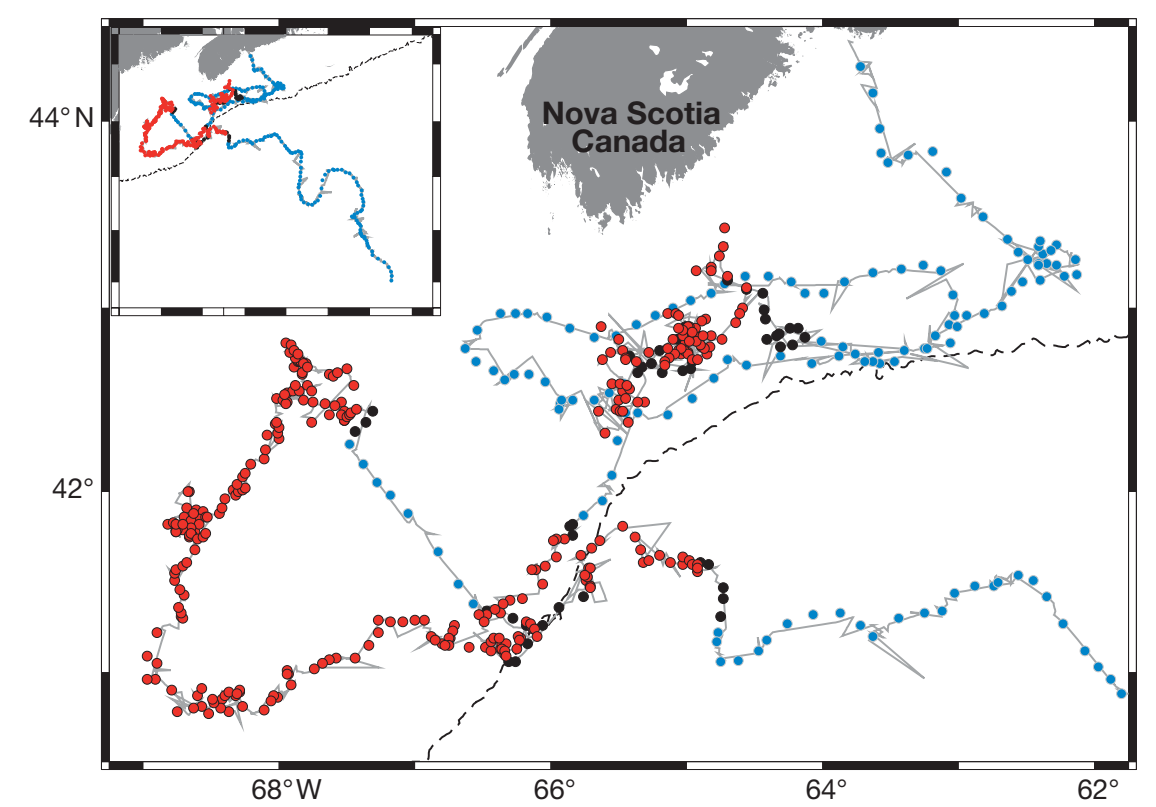

Fig. 1. Dermochelys coriacea. State estimates ( $\mathbf{x}_{t}$ filled circles) with associated behavioural mode estimates (blue $=$ transiting, red $=$ foraging, black $=$ uncertain) obtained from the SSSM for a leatherback turtle (B.1) tagged in coastal waters off Nova Scotia, Canada. The full path is shown inset. The underlying grey line indicates the observed Argos positions. The time interval between each $\mathbf{x}_{t}$ is $6 \mathrm{~h}$. The $1000 \mathrm{~m}$ isobath is displayed as a dashed black line 
periods from transiting to foraging (or vice-versa) or with short intervals (i.e. 12 to $18 \mathrm{~h}$ ) of faster, directed movement embedded within longer foraging bouts. The latter may suggest either the presence of a third behavioural mode or an inability of the model to estimate switches from foraging to transiting back to foraging over such a short time interval. Use of a time step shorter than $6 \mathrm{~h}$ might yield better behavioural mode estimation in these instances, but this may come at the cost of overly conservative estimation of foraging modes due to the scale-dependent nature of the $\theta$ and $\gamma$ parameters.

Marginal posterior distributions of the parameters $\theta_{k}$ and $\gamma_{k}$ showed no overlap between the 2 behavioural modes, suggesting that the modes indeed represent distinct classes of movement (Table A2-1). As would be expected for transiting animals, the median $\theta_{1}$ is near 0 and the median $\gamma_{1}$ is relatively high (see Fig. A2-2 for examples). Foraging animals typically exhibited some form of area-restricted search with large turning angles and relatively slow travel rates (e.g. Fig. 1, red circles). The $\theta_{2}$ medians indicate that turtles tend to reverse their direction frequently (at a $6 \mathrm{~h}$ time scale) while foraging (Table A2-1). The turtles also showed relatively low autocorrelation in their turns and speed (Table A2-1), indicating a lack of persistence in turn angle and travel rate from one time step to the next. Travel rates calculated from the state estimates $\left(\mathbf{x}_{t}\right)$ were also clearly distinguishable between the 2 behavioural modes (e.g. see Fig. A2-3).

The means of the estimated $b_{t}$, which range between 1 ( $1 \leq$ transiting $<1.25)$ and $2(1.75<$ foraging $\leq 2)$, match well with the temporal histograms of the diving statistics. There appears to be close correspondence between switches from one behavioural mode to the other and variation in time at temperature. Temperatures sampled by the turtles were more stable when the model predicted the turtle was foraging (e.g. Turtle B, Fig. 2), suggesting that, at least in northern waters, they forage in a relatively narrow temperature range of approximately 13 to $22^{\circ} \mathrm{C}$ (e.g. Turtle B, Fig. 3). Dive durations followed a similar pattern, in that duration increased with increasing temperature when the model predicted turtles were transiting, but durations tended to have no obvious trend (Fig. 2) and were generally shorter when turtles were predicted to be foraging (Fig. 3). There was reasonable correspondence between changes in the pattern of time spent in deeper water and switches from one behavioural mode to the other. Turtles spent the majority of their time in the upper $65 \mathrm{~m}$ of the water column regardless of behaviour, however, deeper dives were more often associated with transiting behaviour (Fig. 3) but were occasionally observed when turtles were foraging (see Fig. A2-4). Maximum depths attained naturally fol- lowed a similar pattern (Figs. 2 \& 3). Representative plots for Turtle A and a turtle (E.1) with a duty-cycled tag are provided in Appendix 2, Figs A2-4 to A2-7 at www.int-res/articles/suppl/m337p255_app.pdf.

Using behavioural mode estimates and diving data for turtles with duty-cycled and continuously-recording tags, we found clear differences in the means of time at depth, temperature, dive duration, and maximum depth when turtles are transiting versus foraging (Fig. 4). When transiting, turtles on average were $32 \mathrm{~m}$ $( \pm 3.2 \mathrm{SE}, 8 \mathrm{df})$ deeper (Fig. $4 \mathrm{a})$, in water $1.4^{\circ} \mathrm{C}$ $( \pm 0.38 \mathrm{SE}, 8 \mathrm{df})$ warmer (Fig. $4 \mathrm{~b})$, had maximum dives $25.5 \mathrm{~m}( \pm 1.97 \mathrm{SE}, 8 \mathrm{df})$ deeper (Fig. 4c), and had dives $6.6 \mathrm{~min}( \pm 0.98 \mathrm{SE}, 8 \mathrm{df})$ longer (Fig. 4d) than when foraging. The random effects means are also significantly different from 0 under the assumption of a lack of independence among annual foraging bouts of single turtles.

\section{DISCUSSION}

Our state-space approach for identifying foraging sites relies on estimating unobserved behavioural modes by modelling the dynamics of the movement process directly. A key assumption of our approach is that, given sufficient time, animal movement pathways are an integration of more than one behavioural mode. The resulting dynamics, represented by the timeseries of observed positions, can be inherently nonlinear and are therefore best analysed with some form of switching model (Morales et al. 2004, Jonsen et al. 2005). SSSMs allow dynamics to be broken into discrete behavioural modes by specifying a process model for each behaviour and then estimating the probability of switching from one behavioural mode to another at each time step. In our case, the process models for the transiting and foraging behaviours are functionally identical and differ only in the parameter values. This approach also allows for additional complexity such as modelling switches between more than 2 behaviours and modelling the influence of environmental features (Morales et al. 2004) or physiological constraints on switching probabilities.

Search theory predicts that animals will change their behaviour and, consequently, their movement pattern as they encounter changes in habitat or prey density (Turchin 1991). Typically, animals encountering prey at a sufficiently high density will engage in arearestricted search behaviours that are distinct from transiting or migration behaviour. Examination of observed Argos locations shows that leatherbacks engage in both area-restricted search and transiting behaviours; but clear, objective delineation of the change points between behaviours is challenging due 

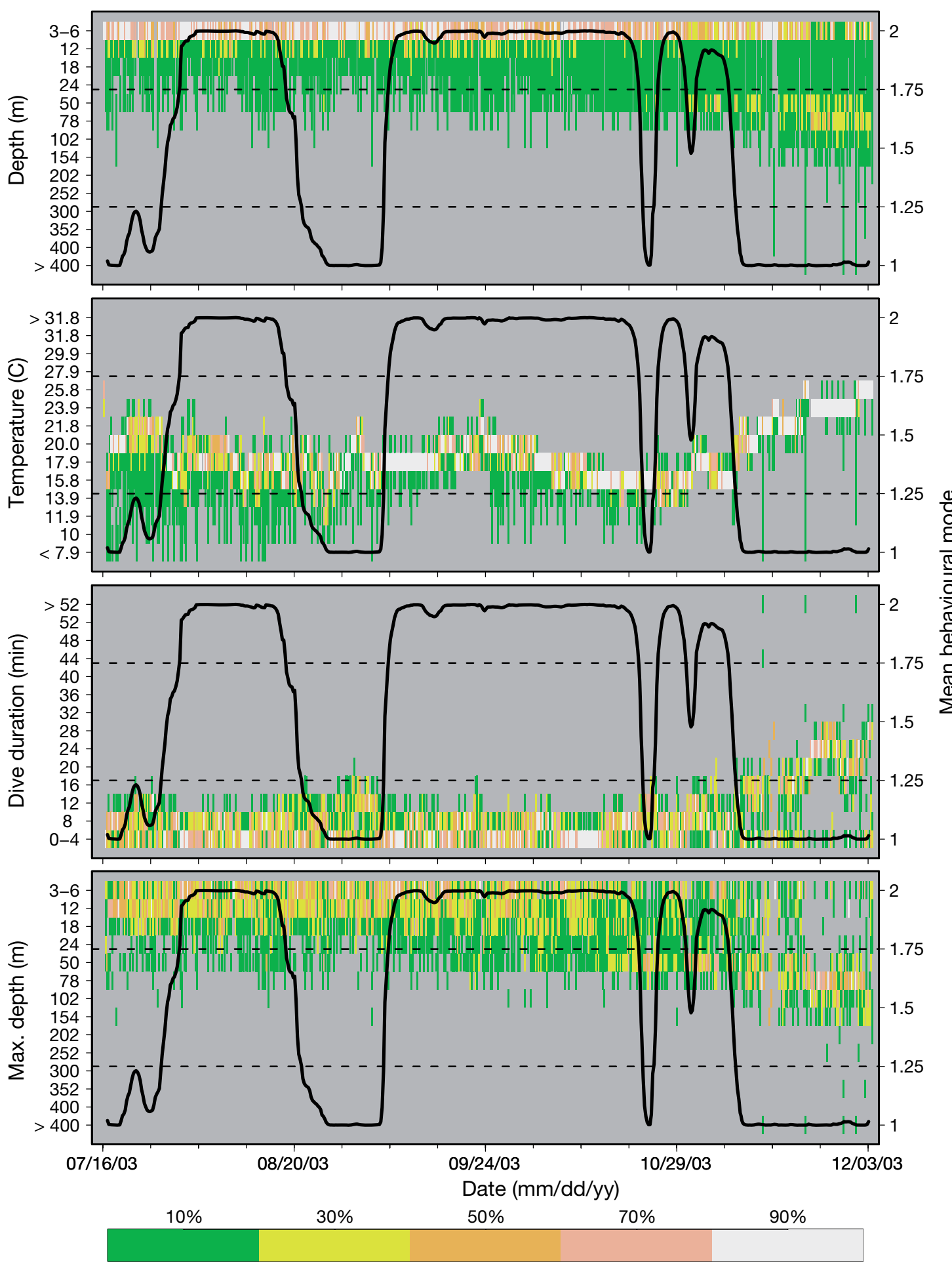

Fig. 2. Dermochelys coriacea. Temporal histograms of time at depth, water temperature, dive duration, and maximum dive depth for turtle B.1 (non-duty-cycled tag). The bins presented on the left vertical axis are the 14 user-defined ranges for each variable. The coloured shingle in each bin indicates percentage of time or dives observed in that range over a $6 \mathrm{~h}$ period. The time periods are: $21: 00-03: 00 \mathrm{~h} ; 03: 00-09: 00 \mathrm{~h} ; 09: 00-15: 00 \mathrm{~h} ;$ 15:00-21:00 h. The labels indicate the mid-point of each bin. Bins shaded grey indicate no dives were observed in that range for that $6 \mathrm{~h}$ time period. Estimated behavioural mode $b_{t}$ for each $6 \mathrm{~h}$ period is overlaid (solid black line). Estimates less than 1.25 (lower dashed line) represent transiting (blue circles in Fig. 1), estimates greater then 1.75 (upper dashed line) represent foraging (red circles in Fig. 1), and estimates between the dashed lines are not assigned to either behavioural mode (the behavioural mode for these time steps could not be estimated with reasonable certainty, i.e. $\left.1.25<b_{t}<1.75\right)$. These uncertain estimates of $b_{t}$ correspond with the black circles in Fig. 1 


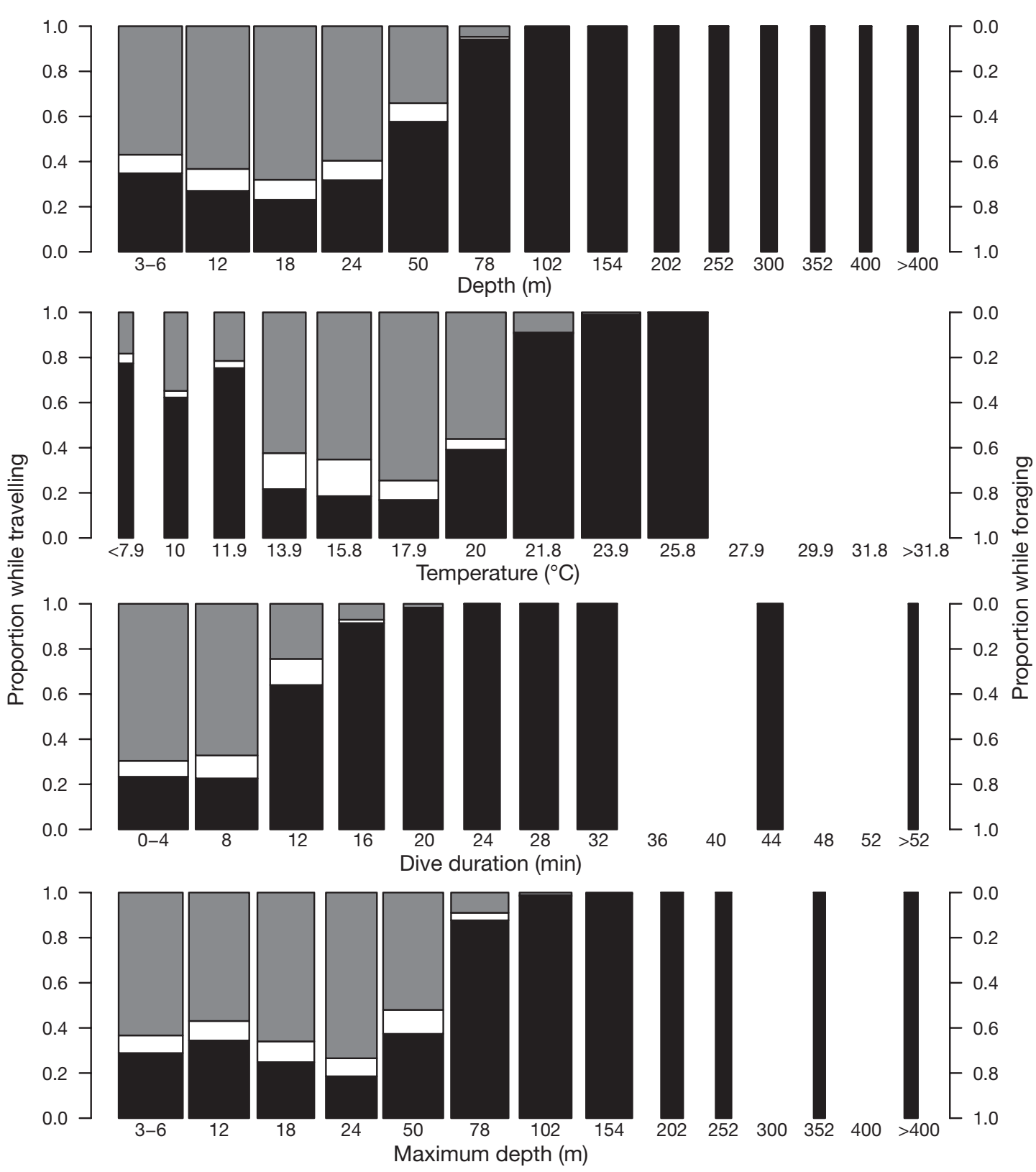

Fig. 3. Dermochelys coriacea. Proportion of observations within each depth, temperature or time bin obtained while turtle B.1 was transiting (black) or foraging (grey). The proportion of observations associated with behavioural modes that could not be estimated with reasonable certainty (see Fig. 2 legend) are displayed in white. The width of each bin is proportional to the total number of observations (all behavioural modes) within that range. The labels indicate the mid-point of each bin. Empty bins indicate no dives were observed in that range for any behavioural state over the duration of the movement pathway. Proportion of observations in each bin while foraging can be read from the top down on the right vertical axis

to the measurement errors in the data. By fitting an SSSM to the 2-dimensional position data we were able to extract the behavioural dynamics obscured by measurement error in the positions. Even though considerable information contained in the third dimension was ignored, the correspondence between behavioural mode predictions and the diving data suggests that behavioural estimation based only on the 2-dimensional position data sufficiently captures the behavioural dynamics. Nevertheless, information on diving behaviour could be incorporated into the SSSM to further refine estimation of behavioural switches and identification of foraging habitat.

Because leatherbacks forage throughout the epipelagic and into the mesopelagic zones on gelatinous zooplankton (James et al. 2005a), we assumed that corresponding changes in horizontal behaviour would be apparent in the vertical movement. Indeed the turtles 

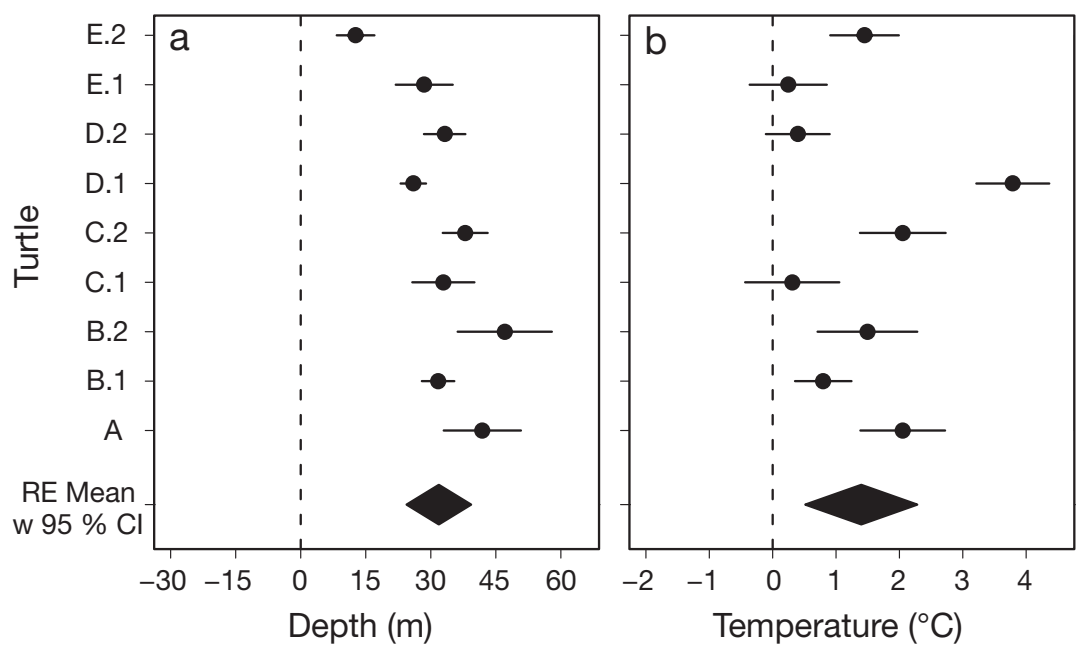

slightly cooler than that associated with waters through which the turtles transit. The cooler waters associated with leatherback foraging may result from thermocline and, perhaps, an associated halocline effect on jellyfish movements (Graham et al. 2001). The narrow range of temperatures experienced while foraging $\left(13\right.$ to $22^{\circ} \mathrm{C}$ ) corresponds well with the average sea surface temperature (SST) $\left(16.6^{\circ} \mathrm{C}\right)$ associated with peak sightings of leatherbacks off of Nova Scotia (James et al. 2006b). Furthermore, a lack of bi-modality in the range of temperatures experienced while foraging suggests that leatherbacks do not cross frontal boundaries frequently, at least in the northern waters considered here (Polovina et al. 2004).

Leatherbacks found off South Africa engage in horizontal movements similar to the foraging and transiting behaviours shown here, but their movements do not correspond to simultaneously collected diving data (Sale et al. 2006). Leatherbacks in this area may be transported by the tremendously strong Agulhas current system (Luschi et al. 2003) and putative switches between transiting and foraging behaviours may simply be the result of advection rather than active behaviour per se. In the presence of strong currents, our model might incorrectly classify the behaviours, particularly when apparent area-restricted search behaviour is due purely to advection. In these situations more detailed models that incorporate information on ocean currents (Gaspar et al. 2006) will be required to reliably identify behavioural states by parsing realized move-

tend to spend more time at greater depths and dive durations are longer when transiting versus foraging. This pattern lends support to the idea that leatherbacks may make scouting dives while transiting as an efficient means for sampling prey density (James et al. 2006a) and perhaps opportunistic foraging without greatly reducing travel rate. Moreover, the shallower dives associated with extended, and presumably more profitable, foraging suggest that the turtles focus on regions where prey are available at shallower strata perhaps to reduce the energetic demands of foraging at depth. The thermal habitat of these foraging areas is ments into passive advection and active behavioural components.

The SSSM approach to identifying multiple behaviours in movement data is more powerful than descriptive methods such as nonlinear curve-fitting to the log-frequency of behavioural events (Sibly et al. 1990), path tortuosity and/or scale-dependency measures (Johnson et al. 2002), and first-passage time analysis (Fauchald \& Tveraa 2003), because it permits the formulation and fitting of mechanistic movement models to the data (Morales et al. 2004). In general, we need models based upon clear statistical principles that 
allow estimation of the dynamical parameters that capture the essence of complex movement behaviour; descriptive methods cannot do this.

Hidden Markov models have recently been used to estimate movement parameters including the probability of switching between different behavioural states (Morales et al. 2004). The Hidden Markov approach is conceptually similar to our SSSM approach with the following 2, albeit related, differences. First, by fitting a switching model in a state-space context we are able to model the dynamics of the movement process directly (Jonsen et al. 2005). Hidden Markov models do not estimate dynamics per se and thus may be unable to capture more complex behavioural processes. Second, observation errors can be accounted for in the Hidden Markov context (Morales et al. 2004), but they cannot be separated from variability in the underlying process that is implicitly being modelled. This drawback of the Hidden Markov approach will likely lead to overestimation of the observation errors, or, if the errors are assumed to be known, may lead to biased movement parameter estimates.

Identification of habitat and habitat use for marine predators is of critical importance for effective conservation and management efforts. Assessment of habitat use is dominated by kernel density estimation methods (Seaman \& Powell 1996) which give each observed position equal weight and ignore underlying behavioural mechanisms that constrain animal movement to particular regions or habitats. Our method can be used to inform kernel density estimation with behavioural information either by considering only those state estimates associated with foraging or by weighting the estimation with the $b_{t}$ estimates (e.g. mean of MCMC samples for each $b_{t}$ ) so that state estimates associated with transiting carry little or no weight. Of course, a more powerful method would be to model directly the relationship between habitat features, such as sea surface temperature or current vectors, and movement behaviour (Jonsen et al. 2003, Morales et al. 2004) to gain a mechanistic understanding of how the animals interact with their environment.

\section{CONCLUSIONS}

Using an SSSM that assumes a first-difference correlated random walk on positions in space and time, we show that distinct classes of movement behaviour can be readily estimated from error-prone Argos telemetry data. Moreover, the switches between behavioural modes correspond with marked changes in the patterns of time at depth, temperature at depth, and dive duration. The correspondence between the model predictions and the diving data is impressive because the
SSSM only makes use of information contained in the time series of positions to estimate the behavioural modes; the model is ignorant of the diving data. Further refinement of the behavioural mode estimation is possible by allowing the model switching to depend on the diving behaviour and/or environmental variables.

The leatherback turtle is one of the most difficult animals to study when they are foraging, furthermore, its jellyfish prey are nearly transparent and are even more difficult to study (but see Witt et al. 2007). Thus, our approach provides insights that cannot be obtained from other methods; but it would be useful to verify the predictions of our models using other independent data, for example, from sensors that detect changes in stomach temperature, mouth opening, and/or animalborne video cameras. However, these methods are difficult to use for long periods of time for an animal like a leatherback turtle, that cannot usually be recaptured.

State-space methods represent a critical advancement in the analysis of electronic tracking data by accounting for uncertainty in the data and by directly modelling the dynamics of animal behaviour. For example, the uncertainty in Argos-derived positions can be an order of magnitude greater than the distances over which foraging animals typically move (Jonsen et al. 2005). In these situations, use of standard methods such as travel rate filters to remove suspect observations will inevitably yield misleading results (Jonsen et al. 2006). Only by explicitly accounting for uncertainty in the data and modelling the dynamic nature of behavioural processes can we gain meaningful insight into the interactions between animals and their environment.

Acknowledgements. We thank J. Baum, B. Block, G. Breed, and S. Jorgensen for helpful comments. Funding was provided from NSERC and Sloan Foundation grants to R.A.M. and from a National Program on Complex Data Systems (NPCDS) grant to J. Mills Flemming, C. Field, M. Dowd, and R. Rutledge. M.C.J. acknowledges support from the U.S. National Marine Fisheries Service, Department of Fisheries and Oceans Canada, Canadian Wildlife Federation, World Wildlife Fund Canada, and Environment Canada. This is a contribution of the Future of Marine Animal Populations (FMAP), part of the Sloan Census of Marine Life. Ransome Myers passed away on 27 March 2007. His brilliance, ethics, and deep concern for the oceans is greatly missed.

\section{LITERATURE CITED}

Bayley GV, Hammersley JM (1946) The 'effective' number of independent observations in an autocorrelated time series. J R Stat Soc 8:184-197

Block BA, Dewar H, Blackwell SB, Williams TD and 7 others (2001) Migratory movements, depth preferences, and thermal biology of Atlantic bluefin tuna. Science 293: 1310-1314

Block BA, Teo SLH, Walli A, Boustany A and 5 others (2005) Electronic tagging and population structure of Atlantic bluefin tuna. Nature 434:1121-1127 
Fauchald P, Tveraa T (2003) Using first-passage time in the analysis of area-restricted search and habitat selection. Ecology 84:282-288

Gaspar P, Georges JY, Fossette S, Lenoble A, Ferraroli S, Maho YL (2006) Marine animal behaviour: neglecting ocean currents can lead us up the wrong track. Proc R Soc B 273:2697-2702

Graham WM, Pages F, Hamner WM (2001) A physical context for gelatinous zooplankton aggregations: a review. Hydrobiologia 451:199-212

IUCN (2004) IUCN red list of threatened species. Available at: www.iucnredlist.org

James MC, Myers RA, Ottensmeyer CA (2005a) Behaviour of leatherback sea turtles, Dermochelys coriacea, during the migratory cycle. Proc R Soc B 272:1547-1555

James MC, Ottensmeyer CA, Myers RA (2005b) Identification of high-use habitat and threats to leatherback sea turtles in northern waters: new directions for conservation. Ecol Lett 8:195-201

James MC, Ottensmeyer CA, Eckert SA, Myers RA (2006a) Changes in diel diving patterns accompany shifts between northern foraging and southward migration in leatherback turtles. Can J Zool 84:754-765

James MC, Sherrill-Mix S, Martin K, Myers RA (2006b) Canadian waters provide critical foraging habitat for leatherback sea turtles. Biol Conserv 133:347-357

James MC, Sherrill-Mix SA, Myers RA (2007) Population characteristics and seasonal migrations of leatherback sea turtles at high latitudes. Mar Ecol Prog Ser 337:245-254

Johnson CJ, Parker KL, Heard DC, Gillingham MP (2002) Movement parameters of ungulates and scale-specific responses to the environment. Ecology 71:225-235

Jonsen ID, Myers RA, Mills Flemming J (2003) Meta-analysis of animal movement using state-space models. Ecology 84:3055-3063

Jonsen ID, Mills Flemming J, Myers RA (2005) Robust statespace modeling of animal movement data. Ecology 86: $2874-2880$

Jonsen ID, Myers RA, James MC (2006) Robust hierarchical state-space models reveal diel variation in travel rates of migrating leatherback turtles. J Anim Ecol 75:1046-1057

Editorial responsibility: Howard Browman (Associate Editorin-Chief), Storebø, Norway
Luschi P, Sale A, Mencacci R, Hughes GR, Lutjeharms JRE, Papi F (2003) Current transport of leatherback sea turtles (Dermochelys coriacea) in the ocean. Proc R Soc B 270: S129-S132

Morales JM, Haydon DT, Friar J, Holsinger KE, Fryxell JM (2004) Extracting more out of relocation data: building movement models as mixtures of random walks. Ecology 85:2436-2445

Newman KB, Buckland ST, Lindley ST, Thomas L, Fernandez C (2006) Hidden process models for animal population dynamics. Ecol Appl 16:74-86

Nielsen A, Bigelow KA, Musyl MK, Sibert JR (2006) Improving light-based geolocation by including sea surface temperature. Fish Oceanogr 15:314-325

Polovina JJ, Balazs GH, Howell EA, Parker DM, Seki MP, Dutton PH (2004) Forage and migration habitat of loggerhead (Caretta caretta) and olive ridley (Lepidochelys olivacea) sea turtles in the central North Pacific Ocean. Fish Oceanogr 13:36-51

Sale A, Luschi P, Mencacci R, Lambardi P, Hughes GR, Hays GC, Benvenuti S, Papi F (2006) Long-term monitoring of leatherback turtle diving behaviour during oceanic movements. J Exp Mar Biol Ecol 328:197-210

Seaman DE, Powell RA (1996) An evaluation of the accuracy of kernel density estimators for home range analysis. Ecology 77:2075-2085

Sibly RM, Nott HMR, Fletcher DJ (1990) Splitting behaviour into bouts. Anim Behav 39:63-69

Spiegelhalter DJ, Thomas A, Best NG, Lunn D (2004) WinBUGS user manual, version 2.0. Medical Research Council Biostatistics Unit, Institute of Public Health, Cambridge

Turchin P (1991) Translating foraging movements in heterogeneous environments into the spatial distribution of foragers. Ecology 72:1253-1266

Witt MJ, Broderick AC, Johns DJ, Martin C, Penrose R, Hoogmoed MS, Godley BJ (2007) Prey landscapes help identify potential foraging habitats for leatherback turtles in the northeast Atlantic. Mar Ecol Prog Ser 337:231-244

Worm B, Myers RA (2003) Meta-analysis of cod-shrimp interactions reveals top-down control in oceanic food webs. Ecology 84:162-173

Submitted: August 25, 2006; Accepted: November 2, 2006 Proofs received from author(s): April 22, 2007 